\title{
Cost-Effectiveness of Insulin Degludec Versus Insulin Glargine U300 in the Netherlands: Evidence From a Randomised Controlled Trial
}

\author{
Marc Evans - Robert G. J. Moes · Katrine S. Pedersen • \\ Jens Gundgaard · Thomas R. Pieber
}

Received: February 28, 2020 / Published online: April 18, 2020

(C) The Author(s) 2020

\begin{abstract}
Introduction: This study aimed to evaluate the short-term cost-effectiveness of insulin degludec 200 units $/ \mathrm{mL}$ (degludec) versus insulin glargine 300 units $/ \mathrm{mL}$ (glargine U300) from a Dutch societal perspective.

Methods: A previously published model estimated costs [2018 euros (EUR)] and effectiveness [quality-adjusted life years (QALYs)] with degludec compared with glargine U300 over a
\end{abstract}

Digital Features To view digital features for this article go to https://doi.org/10.6084/m9.figshare.12046512.

Electronic Supplementary Material The online version of this article (https://doi.org/10.1007/s12325020-01332-y) contains supplementary material, which is available to authorized users.

M. Evans ( $\square)$

University Hospital Llandough, Penarth, UK

e-mail: marclyndon1@hotmail.com

R. G. J. Moes

Novo Nordisk B.V., Alphen aan den Rijn,

Netherlands

K. S. Pedersen

Novo Nordisk Region Europe, Copenhagen,

Denmark

J. Gundgaard

Novo Nordisk A/S, Søborg, Denmark

T. R. Pieber

Department of Internal Medicine, Medical

University of Graz, Graz, Austria 1-year time horizon. The model captured hypoglycaemia rates and insulin dosing. Clinical outcomes were informed by CONCLUDE (NCT03078478), a head-to-head randomised controlled trial in insulin-experienced patients with type 2 diabetes.

Results: Treatment with degludec was associated with mean annual cost savings (EUR 24.71 per patient) relative to glargine U300, driven by a lower basal insulin dose and lower severe hypoglycaemia rate with degludec compared with glargine U300. Lower rates of non-severe nocturnal and severe hypoglycaemia resulted in improved effectiveness (+ 0.0045 QALYs) with degludec relative to glargine U300. In sensitivity analyses, changes to the vast majority of model parameters did not materially affect model outcomes.

Conclusions: This short-term analysis, informed by the latest clinical trial evidence, demonstrated that degludec was a cost-effective treatment option relative to glargine U300. As such, our modelling analysis suggests that degludec would represent an efficient use of Dutch public healthcare resources in this patient population.

Keywords: Diabetes; Health technology assessment; Hypoglycaemia; Insulin analogue; Insulin therapy; Pharmaco-economics 


\section{Key Summary Points}

Why carry out this study?

As the societal burden of chronic diseases continues to rise, economic decision modelling tools are increasingly used to support healthcare decision-making.

As cost-effectiveness is a relative concept, one of the most important structural modelling choices is selection of the most appropriate comparator to ensure that results are relevant and applicable to healthcare decisions.

Here, we evaluated the short-term costeffectiveness of two long-acting basal insulins, degludec U200 (degludec) versus glargine U300, in insulin-experienced patients with type 2 diabetes (T2D) and informed by the latest head-to-head clinical trial evidence (CONCLUDE).

\section{What was learned from this study?}

From a Dutch societal perspective, degludec improved effectiveness at lower cost relative to glargine U300 in insulinexperienced patients with T2D.

As such, our modelling analysis suggests that degludec would represent an efficient use of Dutch public healthcare resources in this patient population.

\section{INTRODUCTION}

Diabetes is associated with a number of debilitating long-term complications, which significantly affect patient quality-of-life and result in substantial morbidity, mortality and healthcare resource utilisation [1-3]. As such, in 2015, diabetes was identified as the 15th most important cause of life-years lost [4].

Economic decision modelling tools are increasingly used to support healthcare decision-making [5]. Cost-effectiveness analyses
(CEAs), which compare the costs of interventions with their outcomes [most commonly the quality-adjusted life year (QALY)], are an important tool to assist healthcare systems with decisions regarding reimbursement for healthcare technologies [6]. As cost-effectiveness is a relative concept, one of the most important structural choices for a CEA is the choice of a comparator; an inappropriate comparator may make the results unsuitable to support healthcare decision-making [7]. The selected comparator should be the most relevant, as informed by current evidence or opinion. In terms of maximising health (QALYs), the most relevant comparator is the 'next best' intervention when ranked by clinical effectiveness [8]. Insulin degludec 200 units/mL (degludec; the degludec 100 units/mL and 200 units/mL formulations are bioequivalent [9]) and insulin glargine 300 units/mL (glargine U300) are two long-acting basal insulin analogues with a lower risk of hypoglycaemia than previously possible with other basal insulin analogues [10]. As such, the authors propose that the two basal insulin analogues, degludec and glargine U300, are highly relevant comparators to inform healthcare decision-making.

The Trial COmpariNg the EffiCacy and Safety of Insulin DegLUDEc and Insulin Glargine 300 Units/mL in Subjects with Type 2 Diabetes Mellitus Inadequately Treated with Basal Insulin and Oral Antidiabetic Drugs (CONCLUDE) was a multinational, head-to-head randomised controlled trial (RCT) that compared the hypoglycaemia risk of degludec with that of glargine U300 in insulin-experienced patients with type 2 diabetes [11]. In CONCLUDE, the primary endpoint, the rate of overall symptomatic hypoglycaemia [defined as severe (an event requiring third-party assistance [12]) or blood glucose $[\mathrm{BG} ;<3.1 \mathrm{mmol} / \mathrm{L} \quad(56 \mathrm{mg} / \mathrm{dL})]$ confirmed events with symptoms], which was tested for superiority, was not significantly different between the two treatment arms during the maintenance period [rate ratio (RR) 95\% confidence interval (CI) 0.88 (0.73; 1.06)] [11]. However, the secondary endpoints, analysed using pre-specified statistical models, showed lower rates of nocturnal symptomatic [events occurring between 00:01 and 05:59; RR: 0.63 
95\% CI $(0.48 ; 0.84)]$ and severe hypoglycaemia [RR $0.2095 \%$ CI $(0.07 ; 0.57)$ ] during the maintenance period with degludec compared with glargine U300 [11].

Healthcare in Europe is provided through a range of different systems at the national level. Since 2005, CEAs have been integral to the Dutch medicine reimbursement system [13]. Currently, the Dutch healthcare system uses a willingness-to-pay threshold range of 20,000 to 80,000 euros (EUR) per QALY gained, depending on disease severity and from a societal perspective (rather than an individual perspective) $[13,14]$.

The aim of the present analysis was to evaluate the short-term cost-effectiveness of degludec compared with glargine U300 in insulinexperienced patients with type 2 diabetes. The analysis was conducted from a Dutch societal perspective and was informed by clinical outcomes from the CONCLUDE trial.

\section{METHODS}

\section{CONCLUDE Trial Design}

The trial design and primary results of CONCLUDE (NCT03078478) have been previously published $[11,15]$. In brief, CONCLUDE was a multinational, randomised, open-label, treat-totarget, active comparator-controlled trial. Eligible patients were adults with type 2 diabetes, who had been previously treated with a basal insulin with or without oral antidiabetic drugs (OADs; any combination of metformin, dipeptidyl peptidase 4 inhibitor, $\alpha$-glucosidase inhibitor, thiazolidinedione and sodium-glucose cotransporter 2 inhibitor) at a stable dose, and who fulfilled at least one pre-defined criterion for hypoglycaemia risk. Patients $(N=1609)$ were randomly assigned 1:1 to receive degludec or glargine U300, both administered once daily with or without OADs. Within each treatment arm, patients were also randomised 1:1 to either morning or evening basal insulin administration. Any pre-trial OADs were continued at their pre-trial dose for the full trial duration, unless safety reasons necessitated a change.
The original 52-week CONCLUDE trial-a 16-week titration period followed by a 36-week maintenance period-was extended following a protocol amendment implemented in February 2018 (described in detail in [15]). Routine monitoring of blinded data identified an unusual pattern in the reporting of glycaemic parameters and hypoglycaemic events, raising patient safety concerns. Therefore, as a result of these concerns, patients were switched from the original glycaemic data collection system (MyGlucoHealth BG meter and electronic diary) to an Abbott BG meter and paper diary, which was used for the remainder of the trial. All patients had completed the 16-week titration period prior to the switch. A new 36-week maintenance period was included in the trial to accommodate these changes, preserve scientific integrity and ensure sufficient data collection for the confirmatory endpoints using the same glycaemic data collection system. On the basis of this new maintenance period, the total treatment period was up to 88 weeks. All analyses of CONCLUDE endpoints in the maintenance period considered the new maintenance period only. CONCLUDE was conducted in accordance with the provisions of the Declaration of Helsinki and the International Conference on Harmonisation Good Clinical Practice Guidelines. The CONCLUDE protocol was approved by the independent ethics committee or institutional review board at each trial centre and written informed consent was obtained from each patient before any trial-related activities.

\section{Cost-Effectiveness Model Overview}

A published, transparent model developed in Microsoft Excel [16] was used to evaluate the short-term cost-effectiveness of degludec compared with glargine U300 in terms of costs (2018 EUR) and QALYs from a Dutch societal perspective. This short-term cohort model with a single annual cycle was informed by data on clinical outcomes from the CONCLUDE trial population, and captured hypoglycaemia rates (non-severe daytime, non-severe nocturnal and severe) and insulin dosing (Fig. 1). The model 


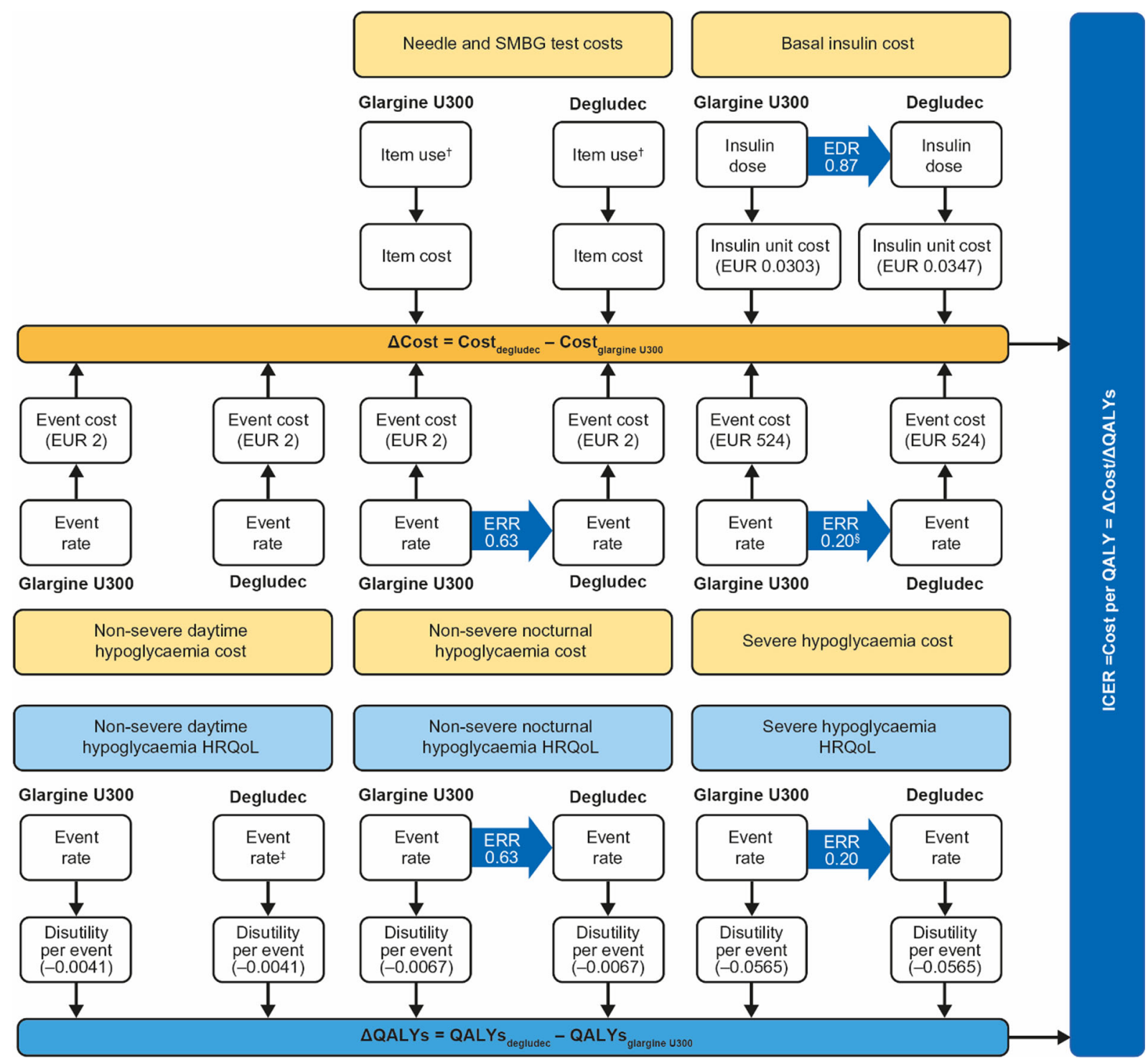

Fig. 1 Cost-effectiveness model schematic. Includes data presented in Tables 1 and 2, in addition to data previously reported [11]. ${ }^{\dagger}$ Assumed to be the same in both simulation arms: one needle and three SMBG tests per day. ${ }^{*}$ The model only used significant treatment effects. As the analysis was non-significant for this endpoint, the glargine U300 event rate was assumed in both simulation arms.

utilised hypoglycaemia data collected in the 36-week maintenance period of CONCLUDE to allow the comparison of hypoglycaemia rates when glycaemic control and insulin dose were stable, and to reduce potential carryover effects from prior treatment regimens.
${ }^{\S}$ Previously reported [11]. $\Delta$ difference in, degludec insulin degludec 200 units $/ \mathrm{mL}, E D R$ estimated dose ratio, $E R R$ estimated rate ratio, EUR euros, glargine U300 insulin glargine 300 units $/ \mathrm{mL}, H R Q O L$ health-related quality of life, ICER incremental cost-effectiveness ratio, QALY quality-adjusted life year, $S M B G$ self-measured blood glucose

\section{Treatment Effects}

Treatment differences (between degludec and glargine U300) were estimated using hypoglycaemia rate and insulin dose ratios from regression analyses of data from the CONCLUDE trial population. To avoid any overlap 
Table 1 Input parameters: clinical outcomes from the CONCLUDE trial and costs and utilities

\begin{tabular}{|c|c|c|c|c|c|c|}
\hline & $\begin{array}{l}\text { Degludec/glargine } \\
\text { U300 ratio }\end{array}$ & SE & 95\% CI & $P$ value & Glargine U300 & Degludec $^{\dagger}$ \\
\hline \multicolumn{5}{|l|}{ Hypoglycaemia } & \multicolumn{2}{|c|}{ Event rate (events/PYO) } \\
\hline Non-severe daytime & $1.09^{\ddagger}$ & 0.1069 & $0.88 ; 1.34$ & 0.4293 & 1.46 & 1.46 \\
\hline Non-severe nocturnal & $0.63^{\ddagger}$ & 0.1430 & $0.48 ; 0.84$ & 0.0015 & 0.93 & 0.59 \\
\hline Severe & $0.20^{\ddagger} \S$ & 0.5354 & $0.07 ; 0.57^{\S}$ & 0.0027 & 0.05 & 0.01 \\
\hline \multicolumn{5}{|l|}{ Insulin dosing } & \multicolumn{2}{|l|}{ Mean dose (U) } \\
\hline Insulin dose & $0.87^{\circ}$ & 0.0273 & $0.82 ; 0.92$ & $<0.0001$ & 73.0 & 63.5 \\
\hline \multicolumn{2}{|l|}{ Parameter } & Mean & Unit & \multicolumn{3}{|l|}{ Source } \\
\hline \multicolumn{7}{|l|}{ Costs } \\
\hline \multicolumn{2}{|l|}{ Degludec ${ }^{\dagger \dagger}$} & 0.0347 & EUR & \multicolumn{3}{|c|}{$Z$-Index 2019 [19] } \\
\hline \multicolumn{2}{|l|}{ Glargine $\mathrm{U} 300^{t+}$} & 0.0303 & EUR & \multicolumn{3}{|c|}{ Z-Index 2019 [19] } \\
\hline \multicolumn{2}{|l|}{ Needle $^{\S \S}$} & 0.1670 & EUR & \multicolumn{3}{|c|}{$Z$-Index 2019 [19] } \\
\hline \multicolumn{2}{|l|}{ SMBG test ${ }^{\text {Tा }}$} & 0.1916 & EUR & \multicolumn{3}{|c|}{$Z$-Index 2019 [19] } \\
\hline \multicolumn{2}{|c|}{ Non-severe hypoglycaemia } & 2 & EUR & \multicolumn{3}{|c|}{ Adapted from de Groot et al. 2018 [21] } \\
\hline \multicolumn{2}{|l|}{ Severe hypoglycaemia } & 524 & EUR & \multicolumn{3}{|c|}{ Adapted from de Groot et al. 2018 [21] } \\
\hline \multicolumn{7}{|l|}{ Utilities } \\
\hline \multicolumn{2}{|l|}{ Base } & 0.9020 & Utility & \multicolumn{3}{|c|}{ Adapted from Freemantle et al. 2013 [33] } \\
\hline \multicolumn{2}{|c|}{ Non-severe daytime hypoglycaemia ${ }^{\dagger \dagger \dagger}$} & -0.0041 & Disutility & \multicolumn{3}{|c|}{ Evans et al. 2013 [34] } \\
\hline \multicolumn{2}{|c|}{ Non-severe nocturnal hypoglycaemia ${ }^{\dagger \dagger \dagger}$} & -0.0067 & Disutility & \multicolumn{3}{|c|}{ Evans et al. 2013 [34] } \\
\hline \multicolumn{2}{|c|}{ Severe hypoglycaemia ${ }^{\dagger \dagger \dagger}$} & -0.0565 & Disutility & \multicolumn{3}{|c|}{ Evans et al. 2013 [34] } \\
\hline
\end{tabular}

Hypoglycaemia costs were inflation-adjusted to 2018 EUR (latest year) using the Consumer Price Index [20]

CI confidence interval, degludec insulin degludec 200 units $/ \mathrm{mL}$, EUR euros, glargine U300 insulin glargine 300 units $/ \mathrm{mL}$, $P Y O$ patient-year of observation, $S E$ standard error, $S M B G$ self-measured blood glucose, $U$ units

$\dagger$ Model inputs for the degludec arm were estimated by applying the degludec/glargine U300 ratio to the corresponding glargine U300 arm value if there was a significant difference between treatments $(p<0.05)$

Rate ratio of hypoglycaemia recorded in the 36-week maintenance period (week 52-week 88)

$\$$ Previously reported [11]

- End-of-trial (week 88) insulin dose ratio $(\mathrm{U} / \mathrm{kg})$

$\dagger^{\dagger}$ Insulin degludec (in FlexTouch ${ }^{\circledR}$ pen) EUR 62.39 for 1800 units

林 Insulin glargine U300 (in Solostar ${ }^{\circledR}$ pen) EUR 40.89 for 1350 units

$\$$ BD MicroFine ${ }^{\mathrm{TM}}$ needles EUR 16.70 for 100 needles

"ศ SMBG test costs based on Glukotest test strips (EUR 4.88 for 50 strips) and OneTouch UltraSoft $®$ lancets (EUR 0.94 for 10 lancets)

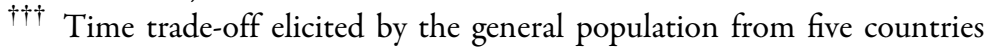


Table 2 Short-term cost-effectiveness of degludec versus glargine U300 in insulin-experienced patients with type 2 diabetes (base case analysis)

\begin{tabular}{lccc}
\hline & Degludec & Glargine U300 & Difference \\
\hline Costs (EUR) & & & -24.71 \\
Total costs & 944.22 & 968.93 & -3.57 \\
Pharmacy costs & 935.01 & 938.58 & -3.57 \\
Insulin $^{\dagger}$ & 804.03 & 807.60 & 0.00 \\
Needles & 61.00 & 61.00 & 0.00 \\
SMBG tests (routine tests) & 69.98 & 69.98 & -21.15 \\
Hypoglycaemic events & 9.21 & 30.35 & 0.00 \\
Non-severe daytime events & 2.92 & 2.92 & -0.69 \\
Non-severe nocturnal events & 1.17 & 1.86 & -20.46 \\
Severe events & 5.11 & 25.57 & \\
QALYs & & & 0.0045 \\
Total QALYs & 0.8915 & 0.8870 & 0.0000 \\
Baseline utility & 0.9020 & 0.9020 & 0.0000 \\
Non-severe daytime hypoglycaemia & -0.0060 & -0.0060 & 0.0023 \\
Non-severe nocturnal hypoglycaemia & -0.0039 & -0.0062 & 0.0022 \\
Severe hypoglycaemia & -0.0006 & -0.0028 & Dominant \\
ICER & & &
\end{tabular}

Difference presented for degludec minus glargine U300. Dominant refers to improved clinical outcomes at a lower cost and is not reported per convention. The disutility associated with hypoglycaemia was captured by multiplying an annualised disutility by the annual event rate

Degludec insulin degludec 200 units $/ \mathrm{mL}$, EUR euros, glargine U300 insulin glargine 300 units $/ \mathrm{mL}$, ICER incremental costeffectiveness ratio, $S M B G$ self-measured blood glucose, $Q A L Y$ quality-adjusted life year

$\dagger$ The insulin dose ratio (in favour of degludec) was factored into the insulin cost calculations

between hypoglycaemia definitions, and therefore the potential to overestimate costs, overall symptomatic hypoglycaemic events in the maintenance period of CONCLUDE were categorised into mutually exclusive groups for modelling purposes and in alignment with other CEAs of degludec (e.g. $[17,18]$ ): non-severe daytime, non-severe nocturnal and severe hypoglycaemia. In the present study, non-severe hypoglycaemia was defined as $\mathrm{BG}<3.1 \mathrm{mmol} / \mathrm{L}(56 \mathrm{mg} / \mathrm{dL})$ with symptoms. Non-severe hypoglycaemic events were categorised as daytime (occurring between 06:00 and 00:00 or the time was unknown), or nocturnal (occurring between 00:01 and 05:59). Severe hypoglycaemia was defined as an event requiring third-party assistance [12].

Analyses of insulin dose, non-severe daytime hypoglycaemia and non-severe nocturnal hypoglycaemia, informed by CONCLUDE, were conducted post hoc; severe hypoglycaemia was analysed as per the pre-specified model in the CONCLUDE trial [11]. Hypoglycaemia endpoints were analysed using a negative binomial model with a log link. The logarithm of the time period in which a hypoglycaemic event was considered treatment emergent as offset. The model included treatment, number of OADs, 
region, gender and dosing time as fixed factors, and age as a covariate. Missing data were imputed by treatment arm using a Poisson model. End-of-trial basal insulin dose (units/kg) was analysed using a mixed model for repeated measures with an unstructured covariance matrix. The model included treatment, number of OADs, region, gender and dosing time as fixed factors; in addition to age, the logarithm of baseline insulin dose and interactions between visit and all exploratory variables as covariates.

Rate and dose ratios from regression analyses were applied to hypoglycaemia rates and insulin dosing observed in the glargine U300 treatment arm in CONCLUDE to derive model inputs for the degludec simulation arm. Treatment effects were only applied where there was a statistically significant difference between treatment arms; otherwise, hypoglycaemia rates and doses from the glargine U300 treatment arm were modelled in both simulation arms (Table 1).

\section{Costs, Utilities and Time Horizon}

Treatment unit costs were based on Dutch list prices (Table 1) [19]. It was assumed that patients administered one basal injection per day, with a new needle and self-measured blood glucose test strip per injection in both simulation arms. Insulin costs were captured as the unit cost multiplied by the mean annual dose. Hypoglycaemia costs and disutilities were derived from the literature, and the costs were inflation-adjusted to 2018 EUR using the Dutch Consumer Price Index (Table 1) [20].

Hypoglycaemia costs were calculated as the sum of costs associated with healthcare, informal care (from informal caregivers) and lost productivity, using data from Dutch patients included in the Global Hypoglycaemia Assessment Tool study and unit costs derived from the Dutch costing manual [21-23]. Costs associated with productivity loss were not included in estimates for severe hypoglycaemia given that the advanced mean baseline age of the CONCLUDE patient population suggests that a large proportion of the patients would be outside the labour market. Hypoglycaemia disutilities were applied by multiplying an annualised disutility by the annual event rate; there were multiple disutilities (for each hypoglycaemia definition). Treatment costs relating to the use of OADs were not included in this analysis as they were assumed to be similar across simulation arms as a result of randomisation and continuation of any pre-trial OADs at the pre-trial dose.

A 1-year time horizon was selected to avoid assumptions regarding the longevity of clinical effects and as a result of the treat-to-target trial design of CONCLUDE, which aimed to achieve glycaemic parity across treatment arms and, thus, obviates the utility of long-term modelling based on differences in glycaemic control. Consequently, the present analysis did not consider the significant, but not clinically relevant, difference in end-of-treatment glycaemic control in favour of degludec (estimated treatment difference $-0.10 \% \quad 95 \%$ CI $[-0.18$; - 0.02]) [11]. The modelled time horizon represents time at maintenance treatment-at relatively stable insulin dose and glycaemic control-and, therefore, does not necessarily represent the first year of treatment and can be extended by additional years, assuming that patients remain at steady state.

\section{Sensitivity Analyses}

One-way deterministic sensitivity analyses were conducted to identify key drivers of outcomes in the base case analysis. Sensitivity analyses explored alternative baseline rates, costs and disutilities for hypoglycaemia. Additional sensitivity analyses were performed varying treatment effects for insulin dosing and hypoglycaemia rates, including those where hypoglycaemia rate ratios were applied regardless of significance and those informed by hypoglycaemia rates observed over the total treatment period of CONCLUDE.

A probabilistic sensitivity analysis (PSA) was conducted to quantify the effect of statistical uncertainty around all relevant stochastic input parameters used in the model. Uncertainty was captured using log-normal distributions (rate and dose ratios) around model parameters, 
informed by standard errors from CONCLUDE analyses (Table 1). PSA outcomes were based on 1000 model iterations sampling from all modelled distributions in each iteration without capturing covariance. For each simulated set of values, an estimate of incremental costs and incremental QALYs was obtained.

\section{RESULTS}

\section{Base Case Analysis}

Treatment with degludec was associated with mean annual total cost savings per patient (EUR 24.71) relative to glargine U300 (Table 2). These cost savings were predominately driven by a lower basal insulin dose with degludec, which more than offset the slightly higher basal insulin unit cost of degludec compared with glargine U300. Cost savings were also driven by a lower rate of severe hypoglycaemia with degludec in comparison with glargine U300. Treatment with degludec was also associated with improved effectiveness (+ 0.0045 QALYs) compared with glargine U300. Quality-of-life benefits with degludec were associated with lower rates of non-severe nocturnal (+ 0.0023 QALYs) and severe hypoglycaemia (+ 0.0022 QALYs) compared with glargine U300 (Table 2). Overall, degludec was a dominant treatment option relative to glargine U300 over 1 year in this patient population.

\section{Sensitivity Analyses}

Degludec was a dominant treatment option relative to glargine U300 in 13 of the 15 oneway deterministic sensitivity analyses demonstrating that the overall cost-effectiveness result is insensitive to variations in most model parameters (Table 3). This was the case for all sensitivity analyses exploring alternative baseline rates, costs and disutilities for hypoglycaemia. For example, the incremental costeffectiveness ratio (ICER) remained dominant in favour of degludec in analyses that applied treatment effects for hypoglycaemia endpoints regardless of their significance or that utilised hypoglycaemia data collected during the total treatment period of CONCLUDE. The ICER was most sensitive to analyses that varied the insulin dose ratio, identifying the lower basal insulin dose requirement with degludec compared with glargine U300 as a key driver of results in the base case analysis. Removing the treatment difference in insulin dose-using the World Health Organization defined daily dose of 40 units of insulin in both simulation armsresulted in an ICER of 9464.00 EUR per QALY gained, which was the most substantial change from the base case result, but remaining well below the Dutch willingness-to-pay lower threshold of 20,000 EUR per QALY gained.

In the PSA, all estimates fell in the eastern quadrants, demonstrating an improvement in QALYs with degludec compared with glargine U300 (Fig. 2a). Furthermore, most estimates were located in the south-east quadrant, indicating that, in addition to improved effectiveness, there were also lower costs with degludec when compared with glargine U300 treatment. Overall, the PSA showed that degludec is likely to be cost-effective with an estimated $100.0 \%$ probability for a willingness-to-pay threshold of 20,000 EUR per QALY gained (Fig. 2b).

\section{DISCUSSION}

Our short-term modelling analysis, from a Dutch societal perspective, suggests that degludec is a cost-effective treatment option relative to glargine U300 in insulin-experienced patients with type 2 diabetes. The overall result was robust in the sensitivity analyses, which indicated that the lower insulin dose requirement with degludec was a key driver of outcomes in the base case analysis.

Our findings expand on the primary findings of the CONCLUDE trial and demonstrate that the lower rates of nocturnal and severe symptomatic hypoglycaemia, in addition to the lower insulin dose requirement reported with degludec relative to glargine $\mathrm{U} 300$, translate into QALY gains with degludec at a lower healthcare cost in this patient population and, hence, an efficient use of Dutch public healthcare resources. 
Table 3 Results of the deterministic sensitivity analyses

\begin{tabular}{|c|c|c|c|}
\hline & $\begin{array}{l}\Delta \text { Costs } \\
(\text { EUR })\end{array}$ & $\Delta Q A L Y s$ & $\begin{array}{l}\text { ICER (EUR per } \\
\text { QALY) }\end{array}$ \\
\hline Base case & -24.71 & 0.0045 & Dominant \\
\hline \multicolumn{4}{|l|}{ Baseline hypoglycaemia rates } \\
\hline UKHSG-T2D $<2$ years & -46.66 & 0.0085 & Dominant \\
\hline UKHSG-T2D > $>$ years & -299.94 & 0.0415 & Dominant \\
\hline Östenson et al. 2014 [35] & -51.44 & 0.0245 & Dominant \\
\hline \multicolumn{4}{|l|}{ Hypoglycaemia rate ratios (RRs) } \\
\hline $\begin{array}{l}\text { CONCLUDE maintenance period (including non-significant } \\
\text { RRs) }\end{array}$ & -24.45 & 0.0040 & Dominant \\
\hline CONCLUDE total treatment period (only significant RRs) ${ }^{\dagger}$ & -20.99 & 0.0035 & Dominant \\
\hline $\begin{array}{l}\text { CONCLUDE total treatment period (including non-significant } \\
\text { RRs) }{ }^{\dagger}\end{array}$ & -21.17 & 0.0039 & Dominant \\
\hline \multicolumn{4}{|l|}{ Insulin dose ratio (DR) } \\
\hline Lower 95\% CI of CONCLUDE end-of-trial insulin DR & -70.92 & 0.0045 & Dominant \\
\hline Upper 95\% CI of CONCLUDE end-of-trial insulin DR & 21.50 & 0.0045 & 4761.05 \\
\hline $\begin{array}{l}\text { WHO DDD (40 units/day in both simulation arms: DR = 1) } \\
\text { [36] }\end{array}$ & 42.73 & 0.0045 & 9464.00 \\
\hline \multicolumn{4}{|l|}{ Hypoglycaemia costs } \\
\hline Increased by $10 \%$ & -26.83 & 0.0045 & Dominant \\
\hline Decreased by $10 \%$ & -22.60 & 0.0045 & Dominant \\
\hline \multicolumn{4}{|l|}{ Hypoglycaemia disutilities } \\
\hline Currie et al. 2006 [37] & -24.71 & 0.0017 & Dominant \\
\hline Lauridsen et al. 2014 [38] & -24.71 & 0.0052 & Dominant \\
\hline $\begin{array}{l}\text { Dutch-specific utilities (non-severe: }-0.0029 \text {; severe: }-0.0097 \text { ) } \\
\text { [23] }\end{array}$ & -24.71 & 0.0014 & Dominant \\
\hline
\end{tabular}

$\triangle$ Costs and $\triangle$ QALYs reported for degludec minus glargine U300. Dominant refers to improved clinical outcomes at a lower cost and is not reported per convention

$\Delta$ difference in, $C I$ confidence interval, $D D D$ defined daily dose, degludec insulin degludec 200 units $/ \mathrm{mL}, D R$ dose ratio, EUR euros, ICER incremental cost-effectiveness ratio, glargine $U 300$ insulin glargine 300 units/mL, QALY quality-adjusted life year, $R R$ rate ratio, T2D type 2 diabetes, UKHSG United Kingdom Hypoglycaemia Study Group, WHO World Health Organization

$\dagger$ Based on clinical model inputs presented in Table S1 in the supplementary material

* Reported per event and adjusted to an annual rate

Prior to the availability of head-to-head data comparing clinical outcomes with degludec compared with glargine U300, a short-term modelling analysis from the perspective of National Health England conducted scenario analyses to evaluate comparative cost- 

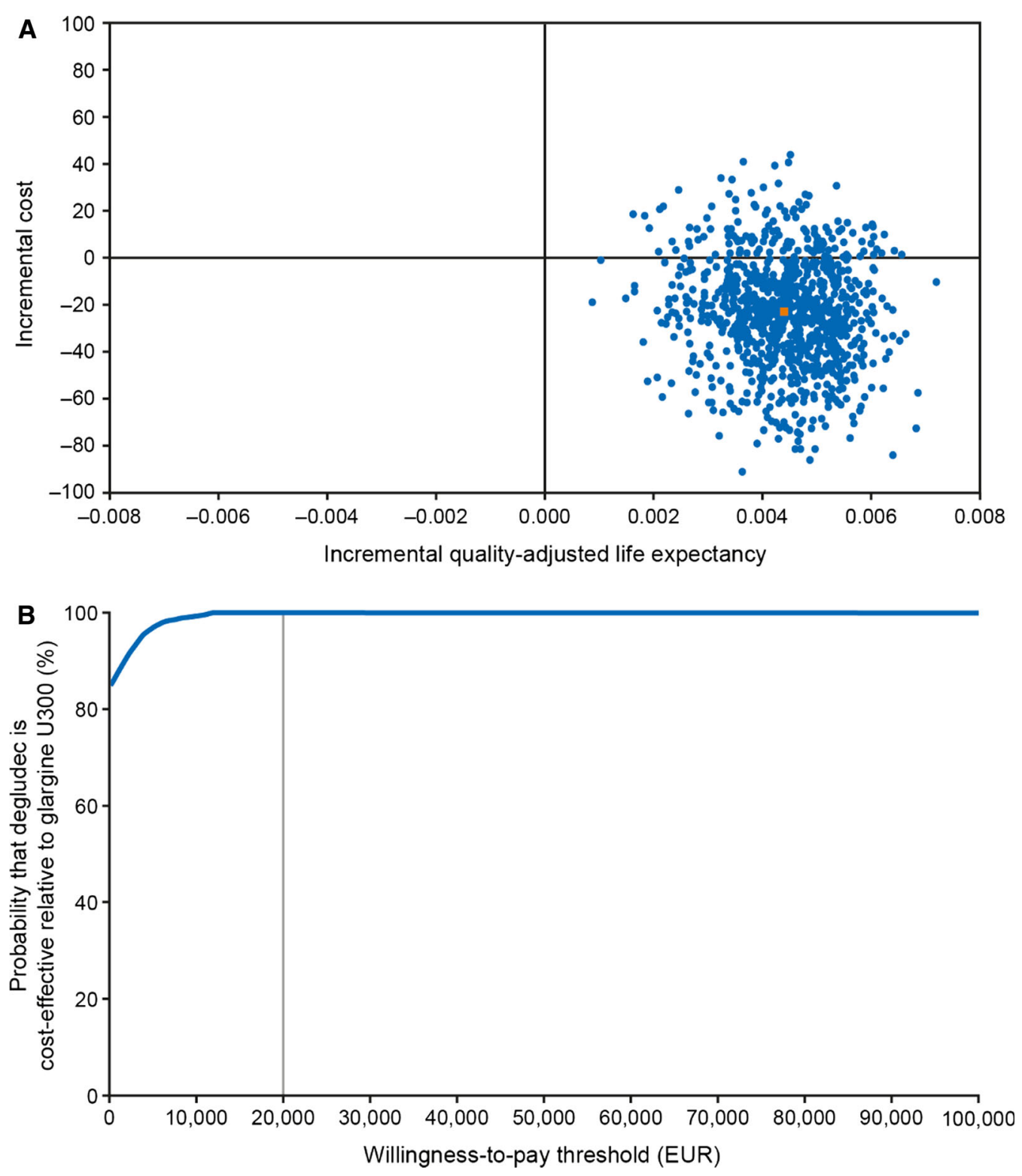

Fig. 2 Probabilistic sensitivity analysis results: a costeffectiveness scatter plot; $\mathbf{b}$ cost-effectiveness acceptability curve. In a, the orange square represents the average value for incremental cost and incremental quality-adjusted life

effectiveness, utilising a number of hypothesisgenerating assumptions [24]. In their analysis, Evans et al. found that degludec was likely to be a dominant treatment option relative to glargine U300 in basal insulin-experienced patients with type 2 diabetes [24]. The results of the present analysis, informed by head-to-head RCT data, are in alignment with those simulated by expectancy. EUR euros, degludec insulin degludec 200 units $/ \mathrm{mL}$, glargine $\mathrm{U} 300$ insulin glargine 300 units $/ \mathrm{mL}$, QALY quality-adjusted life year

Evans et al. [24], albeit from a different perspective and setting.

As with all modelling studies, it is important to consider the limitations when putting the findings into context. For instance, in modelling studies it is assumed that data collected in RCTs are replicated in real-world clinical practice, which may not be the case. CONCLUDE 
was a treat-to-target trial where insulin doses were titrated to a strict glycaemic target, whereas optimal glycaemic control may not be achieved in real-world patients for numerous reasons including clinical inertia, non-adherence and fear of hypoglycaemia [25-27]. However, CONCLUDE enrolled a large cohort of insulin-experienced patients with a long duration of diabetes and risk factors for hypoglycaemia, who represent a more accurate reflection of patients in real-world clinical practice than most insulin RCTs, where patients with hypoglycaemia risk factors are typically excluded (e.g. [28]). It is also important to note that the analyses conducted for the present CEA were mostly post hoc, in order to extract the most relevant data (in alignment with previous CEAs of degludec), and that the different types of hypoglycaemic events analysed (non-severe daytime, non-severe nocturnal, severe) have distinct implications for both costs and QALYs.

The main strengths of this analysis are its simplicity and transparency: clinical outcomes and patient characteristics were taken from a single, large, multinational RCT, while the base case analysis only utilised clinical endpoints with significant treatment effects. Type 2 diabetes is a chronic, progressive disease that requires individualised treatment regimens, with evidence of clinical inertia and heterogeneous treatment algorithms in real-world clinical practice $[29,30]$. Accordingly, our shortterm analysis, which did not rely on assumptions regarding the persistence of treatment effects over long timescales, may represent a suitable perspective to assess maintenance treatment at relatively stable insulin dose and glycaemic control. Alternatively, our analysis could be conducted from a long-term perspective to project the development of diabetes-related complications and mortality, with clinical model inputs informed by surrogate risk factors (e.g. $\mathrm{HbA}_{1 \mathrm{c}}$ ) using an established model such as the IQVIA CORE Diabetes Model [31] or the IHE Cohort Model of Type 2 Diabetes [32]. However, we selected a short-term perspective as a result of the treat-to-target trial design of CONCLUDE and therefore the small treatment differences in risk factors including $\mathrm{HbA}_{1 \mathrm{c}}$, in addition to considerations regarding the uncertainty of risk factor progression and treatment intensification in patients over long timescales. As such, we would expect projected long-term costs and effects (related to diabetes-related complications and mortality) to be similar across simulation arms and not significantly influence the ICER.

In contrast with other degludec CEAs (e.g. [18]), we utilised data for clinical outcomes from the trial maintenance period (rather than the total treatment period) and, therefore, our results are relevant to these treatment conditions i.e. they do not have to represent the first year of treatment and the time horizon can be extended or reduced, as applicable. We explored the influence of utilising data from the total treatment period in a sensitivity analysis, and the overall result was unchanged: degludec was a dominant treatment over 1 year relative to glargine U300.

\section{CONCLUSION}

This short-term cost-effectiveness analysis, informed by the latest RCT evidence, demonstrates that degludec is a dominant treatment option in comparison with glargine U300 from a Dutch societal perspective, resulting in improved clinical outcomes at a lower cost in insulin-experienced patients with type 2 diabetes. As such, our modelling analysis suggests that degludec would represent an efficient use of Dutch healthcare resources in this patient population.

\section{ACKNOWLEDGEMENTS}

We thank the patients, staff and investigators for their participation in CONCLUDE. The authors thank Ehsan Parvaresh Rizi (Novo Nordisk A/S, Denmark) for his review of and input to the manuscript.

Funding. Sponsorship for CONCLUDE, this analysis, the Rapid Service and Open Access Fees were funded by Novo Nordisk A/S. 
Medical Writing Assistance. Medical writing and submission support were provided by Anna Campbell and Germanicus HansaWilkinson of Watermeadow Medical, an Ashfield company, funded by Novo Nordisk.

Authorship. All named authors meet the International Committee of Medical Journal Editors (ICMJE) criteria for authorship for this article, take responsibility for the integrity of the work as a whole, and have given their approval for this version to be published. Marc Evans is the guarantor of this work and, as such, had full access to all the data in the study, and takes responsibility for the integrity of the data and the accuracy of the data analysis.

Prior Presentation. Parts of this analysis were presented as a poster presentation at the meeting of the International Society for Pharmacoeconomics and Outcomes Research Europe 2019, 2-6 November 2019, Copenhagen, Denmark.

Disclosures. Marc Evans has received honoraria and research awards from Novo Nordisk, Sanofi-Aventis, MSD and Novartis. Robert G.J. Moes, Katrine Skipper Pedersen and Jens Gundgaard are employees of Novo Nordisk. Jens Gundgaard and Katrine Skipper Pedersen also hold shares/stocks in Novo Nordisk. Thomas R. Pieber has received research support from Novo Nordisk and AstraZeneca (paid directly to the Medical University of Graz); personal fees as a consultant from AstraZeneca, Bristol-Myers Squibb, Eli Lilly, Novo Nordisk and Roche Diabetes Care. Thomas R. Pieber is also the Chief Scientific Officer of CBmed (Center for Biomarker Research in Medicine), a public-funded biomarker research company.

\section{Compliance with Ethics Guideli-} nes. CONCLUDE was conducted in accordance with the provisions of the Declaration of Helsinki and the International Conference on Harmonisation Good Clinical Practice Guidelines. The CONCLUDE protocol was approved by the independent ethics committee or institutional review board at each trial centre and written informed consent was obtained from each patient before any trial-related activities.

Data Availability. The datasets generated and/or analysed during the current study are available from the corresponding author on reasonable request.

Open Access. This article is licensed under a Creative Commons Attribution-NonCommercial 4.0 International License, which permits any non-commercial use, sharing, adaptation, distribution and reproduction in any medium or format, as long as you give appropriate credit to the original author(s) and the source, provide a link to the Creative Commons licence, and indicate if changes were made. The images or other third party material in this article are included in the article's Creative Commons licence, unless indicated otherwise in a credit line to the material. If material is not included in the article's Creative Commons licence and your intended use is not permitted by statutory regulation or exceeds the permitted use, you will need to obtain permission directly from the copyright holder.To view a copy of this licence, visit http://creativecommons.org/licenses/by$\mathrm{nc} / 4.0 /$.

\section{REFERENCES}

1. Hayes A, Arima H, Woodward M, et al. Changes in quality of life associated with complications of diabetes: results from the ADVANCE study. Value Health. 2016;19:36-41.

2. Chapman D, Foxcroft R, Dale-Harris L, Ronte H, Bidgoli F, Bellary S. Insights for care: the healthcare utilisation and cost impact of managing type 2 diabetes-associated microvascular complications. Diabetes Ther. 2019;10:575-85.

3. American Diabetes Association. Economic costs of diabetes in the US in 2017. Diabetes Care. 2018;41: 917-28.

4. GBD 2015 Mortality and Causes of Death Collaborators. Global, regional, and national life expectancy, all-cause mortality, and cause-specific mortality for 249 causes of death, 1980-2015: a systematic analysis for the Global Burden of Disease Study 2015. Lancet. 2016;388:1459-544. 
5. Caro JJ, Briggs AH, Siebert U, Kuntz KM. Modeling good research practices-overview: a report of the ISPOR-SMDM modeling good research practices task force-1. Value Health. 2012;15:796-803.

6. Robinson R. Cost-utility analysis. BMJ. 1993;307: 859-62.

7. Ziouani S, Granados D, Borget I. How to select the best comparator? An international economic evaluation guidelines comparison. Value Health. 2016;19:A471-A472.

8. Karlsson G, Johannesson M. The decision rules of cost-effectiveness analysis. Pharmacoeconomics. 1996;9:113-20.

9. Korsatko S, Deller S, Koehler G, et al. A comparison of the steady-state pharmacokinetic and pharmacodynamic profiles of 100 and $200 \mathrm{U} / \mathrm{mL}$ formulations of ultra-long-acting insulin degludec. Clin Drug Investig. 2013;33:515-21.

10. Tibaldi JM. Evolution of insulin: from human to analog. Am J Med. 2014;127:S25-38.

11. Philis-Tsimikas A, Klonoff DC, Khunti KB, et al. Risk of hypoglycaemia with degludec versus glargine U300 in insulin-treated patients with type 2 diabetes: the randomised, head-to-head CONCLUDE trial. Diabetologia. 2020;63:698-710.

12. Seaquist ER, Anderson J, Childs B, et al. Hypoglycemia and diabetes: a report of a workgroup of the American Diabetes Association and the Endocrine Society. J Clin Endocrinol Metab. 2013;98: 1845-59.

13. van Baal P, Perry-Duxbury M, Bakx P, Versteegh M, van Doorslaer E, Brouwer W. A cost-effectiveness threshold based on the marginal returns of cardiovascular hospital spending. Health Econ. 2019;28: $87-100$.

14. Brouwer $\mathrm{W}$, van Baal $\mathrm{P}$, van Exel J, Versteegh $\mathrm{M}$. When is it too expensive? Cost-effectiveness thresholds and health care decision-making. Eur J Health Econ. 2019;20:175-80.

15. Philis-Tsimikas A, Stratton I, Norgard Troelsen L, Anker Bak B, Leiter LA. Efficacy and safety of degludec compared to glargine 300 units/mL in insulin-experienced patients with type 2 diabetes: trial protocol amendment (NCT03078478). J Diabetes Sci Technol. 2019;13:498-506.

16. Evans M, Wolden M, Gundgaard J, Chubb B, Christensen T. Cost-effectiveness of insulin degludec compared with insulin glargine in a basal-bolus regimen in patients with type 1 diabetes mellitus in the UK. J Med Econ. 2015;18:56-68.
17. Evans M, Wolden M, Gundgaard J, Chubb B, Christensen T. Cost-effectiveness of insulin degludec compared with insulin glargine for patients with type 2 diabetes treated with basal insulinfrom the UK health care cost perspective. Diabetes Obes Metab. 2014;16:366-75.

18. Evans M, Mehta R, Gundgaard J, Chubb B. Costeffectiveness of insulin degludec vs insulin glargine U100 in type 1 and type 2 diabetes mellitus in a UK setting. Diabetes Ther. 2018;9:1919-30.

19. Intermediair in zorginformatie op maat. Z-Index 2019 https://www.zindex.nl/english. Accessed 28 Feb 2020.

20. Statistics Netherlands. Statline-Consumer Price Index 2019. https://opendata.cbs.nl/statline\#/CBS/ $\mathrm{nl} /$ dataset/83131NED/table?ts=1567177692885.

Accessed 28 Feb 2020.

21. de Groot S, Enters-Weijnen CF, Geelhoed-Duijvestijn PH, Kanters TA. A cost of illness study of hypoglycaemic events in insulin-treated diabetes in the Netherlands. BMJ Open. 2018;8:e019864.

22. Khunti K, Alsifri S, Aronson R, et al. Rates and predictors of hypoglycaemia in 27585 people from 24 countries with insulin-treated type 1 and type 2 diabetes: the global HAT study. Diabetes Obes Metab. 2016;18:907-15.

23. Hakkaart-van Roijen L, Van der Linden N, Bouwmans CAM, Kanters T, Tan SS. Costing manual: methodology of costing research and reference prices for economic evaluations in healthcare 2015. https://www.imta.nl/costingtool/. Accessed 28 Feb 2020.

24. Evans M, Chubb B, Gundgaard J. Cost-effectiveness of insulin degludec versus insulin glargine in adults with type 1 and type 2 diabetes mellitus. Diabetes Ther. 2017;8:275-91.

25. Peyrot M, Barnett AH, Meneghini LF, SchummDraeger PM. Insulin adherence behaviours and barriers in the multinational global attitudes of patients and physicians in insulin therapy study. Diabet Med. 2012;29:682-9.

26. Khunti K, Wolden ML, Thorsted BL, Andersen M, Davies MJ. Clinical inertia in people with type 2 diabetes: a retrospective cohort study of more than 80,000 people. Diabetes Care. 2013;36:3411-7.

27. Fidler C, Elmelund Christensen T, Gillard S. Hypoglycemia: an overview of fear of hypoglycemia, quality-of-life, and impact on costs. J Med Econ. 2011;14:646-55.

28. Ritzel R, Roussel R, Bolli GB, et al. Patient-level meta-analysis of the EDITION 1, 2 and 3 studies: 
glycaemic control and hypoglycaemia with new insulin glargine $300 \mathrm{U} / \mathrm{ml}$ versus glargine $100 \mathrm{U} / \mathrm{ml}$ in people with type 2 diabetes. Diabetes Obes Metab. 2015;17:859-67.

29. Khunti K, Gomes MB, Pocock S, et al. Therapeutic inertia in the treatment of hyperglycaemia in patients with type 2 diabetes: a systematic review. Diabetes Obes Metab. 2018;20:427-37.

30. American Diabetes Association. 9. Pharmacologic approaches to glycemic treatment: Standards of medical care in diabetes-2019. Diabetes Care. 2019;42:90-102.

31. Palmer AJ, Roze S, Valentine WJ, et al. The CORE diabetes model: projecting long-term clinical outcomes, costs and cost-effectiveness of interventions in diabetes mellitus (types 1 and 2) to support clinical and reimbursement decision-making. Curr Med Res Opin. 2004;20(Suppl 1):S5-26.

32. Lundqvist A, Steen Carlsson K, Johansen P, Andersson E, Willis M. Validation of the IHE cohort model of type 2 diabetes and the impact of choice of macrovascular risk equations. PLoS One. 2014;9: e110235.

33. Freemantle N, Evans M, Christensen T, Wolden ML, Bjorner JB. A comparison of health-related quality of life (health utility) between insulin degludec and insulin glargine: a meta-analysis of phase 3 trials. Diabetes Obes Metab. 2013;15:564-71.

34. Evans M, Khunti K, Mamdani M, et al. Health-related quality of life associated with daytime and nocturnal hypoglycaemic events: a time trade-off survey in five countries. Health Qual Life Outcomes. 2013;11:90.

35. Ostenson CG, Geelhoed-Duijvestijn P, Lahtela J, Weitgasser R, Markert Jensen M, Pedersen-Bjergaard U. Self-reported non-severe hypoglycaemic events in Europe. Diabet Med. 2014;31:92-101.

36. WHO Collaborating Centre for Drug Statistics Methodology. ATC/DDD Index 2019. https://www. whocc.no/atc_ddd_index/. Accessed 28 Feb 2020.

37. Currie CJ, Morgan CL, Poole CD, Sharplin P, Lammert M, McEwan P. Multivariate models of healthrelated utility and the fear of hypoglycaemia in people with diabetes. Curr Med Res Opin. 2006;22: $1523-34$.

38. Lauridsen JT, Lønborg J, Gundgaard J, Jensen HH. Diminishing marginal disutility of hypoglycaemic events: results from a time trade-off survey in five countries. Qual Life Res. 2014;23:2645-50. 